\title{
Fault Recovery Based on Checkpointing for Hard Real-Time Embedded Systems*
}

\author{
Ying Zhang and Krishnendu Chakrabarty \\ Department of Electrical \& Computer Engineering, Duke University \\ Email: \{yingzh, krish\}@ee.duke.edu
}

\begin{abstract}
Safety-critical embedded systems often operate in harsh environmental conditions that necessitate fault-tolerant computing techniques. Many safety-critical systems also execute realtime applications. The correctness of these systems depends not only on the logical result of computation, but also on the time at which the results are produced. The missing of task deadlines can therefore be viewed as a temporal fault. In this paper, we examine fault recovery based on checkpointing for real-time systems. We present schedulability tests for checkpointing in real-time systems. These feasibility-of-scheduling tests provide the criteria under which checkpointing can provide fault tolerance and real-time guarantees for hard real-time embedded systems under two different fault arrival models.
\end{abstract}

\section{Introduction}

Embedded systems often operate in harsh environmental conditions that necessitate the use of fault-tolerant computing techniques. Many embedded systems also execute real-time applications that require strict adherence to task deadlines [1]. The correctness of these systems depends not only on the logical result of computation, but also on the time at which the results are produced [2]. The missing of task deadlines can therefore be viewed as a temporal fault. In this paper, we present an integrated approach that provides fault tolerance and real-time responsiveness for tasks executing in an embedded system.

Fault tolerance is typically achieved in real-time systems through a combination of on-line fault detection [3], and checkpointing and rollback recovery [4]. At each checkpoint, the system saves its state in a secure device. When a fault is detected, the system rolls back to the most recent checkpoint and resumes normal execution.

Checkpointing increases task execution time and in the absence of faults, it might cause a missed deadline for a task that completes on time without checkpointing. In the presence of faults however, checkpointing can increase the likelihood of a task completing on time with the correct result. The checkpointing interval, i.e., duration between two consecutive checkpoints, must be carefully chosen to balance checkpointing cost (the time needed to perform a single checkpoint) with the rollback time.

There are three main reasons for incorporating fault tolerance in real-time embedded systems. The first reason is motivated by the rapid increase in processor speeds. Higher processor speeds lead to higher power consumption and heat dissipation; increased die temperatures create thermal stress on the die and undermine system reliability. This results in

\footnotetext{
* This research is sponsored by the Defense Advance Research Projects Agency (DARPA), and administered by the Army Research Office under Emergent Surveillance Plexus MURI Award No. DAAD19-01-1-0504. Any opinions, findings, and conclusions or recommendations expressed in this publication are those of the authors and do not necessarily reflect the views of the sponsoring agencies.
} 
more transient faults during system operation. In order to mitigate reliability problems caused by high die temperatures, we can adopt fault tolerance techniques such as checkpointing.

The second reason is motivated by the need to meet task deadlines in real-time systems. The operational correctness of a real-time system depends on the timely completion of the underlying tasks. Fault tolerance in this scenario can also be viewed as a means to exploit the available slack during task execution. It is well known that checkpointing provides an effective method to save re-execution time in the presence of faults. Hence checkpointing can be used to increase the likelihood of timely task completion in the presence of faults in real-time systems.

The third motivation arises from shrinking process technologies in the nanotechnology realm. Lower processor voltages are likely to lead to lower noise margins and more transient faults, caused in part by single-event upsets [5]. Hence system-level fault tolerance, are of particular interest for such embedded systems.

We assume throughout that faults are intermittent or transient in nature, and that permanent faults are handled through manufacturing testing or field-testing techniques [6]. Typical examples of transient faults include errors caused by cosmic rays and high-energy particles in nanotechnology with shrinking processes [5].

We address hard real-time systems in this paper. Systems in which a missed deadline results in disastrous consequences are termed hard real-time systems [7]. Therefore, it is important to guarantee timeliness for hard real-time systems under worst-case scenarios. In this work, the off-line feasibility analysis is targeting at providing deterministic timeliness for hard real-time systems. Checkpointing for soft real-time systems, in which missed deadlines do not lead to catastrophic consequences, was recently presently in [8].

We present feasibility tests for checkpointing schemes that use a fixed checkpointing interval for real-time tasks. These feasibility tests provide the criteria under which checkpointing can provide fault tolerance and real-time guarantees under two different transient fault arrival models. We also present two techniques to determine the fixed checkpointing interval in an off-line manner.

\section{Checkpointing in real-time systems}

An off-line checkpointing scheme determines the checkpointing interval for a task a priori, i.e., before task execution. Most known checkpointing schemes for real-time systems belong to this category $[9,10,11]$. A drawback here is that the checkpointing interval cannot be adapted to the actual fault occurrence during task execution. An on-line scheme in which the checkpointing interval can be adapted to fault occurrences is therefore more desirable. However, current on-line checkpointing schemes [12] provide only probabilistic guarantees on the timely completion of tasks.

Some checkpointing schemes, e.g. $[9,10]$, assume that faults occur as a Poisson process with arrival rate $\lambda$. These schemes use a checkpointing interval that maximizes the probability that a task completes on time for a given fault arrival rate $\lambda$. Hence the real-time guarantees in these schemes are probabilistic. Other checkpointing schemes, e.g., [11], offer deterministic real-time guarantees under different assumptions. For example, it is sometimes assumed that at most $k$ faults occur during task execution.

Equidistant checkpointing relies on the use of a constant checkpointing interval. This is typically used with off-line checkpointing schemes. If the checkpointing cost is $C$ and faults arrive as a Poisson process with rate $\lambda$, the mean execution time for the task is minimum if a constant checkpointing interval of $\sqrt{2 C / \lambda}$ is used [10]. However, the minimum execution time does not guarantee timely completion of a task under real-time deadlines. 


\section{Off-line feasibility analysis for checkpointing in hard real-time tasks}

In this section, we investigate deterministic fault tolerance for hard real-time tasks under the worst scenario. We are given a set $\Gamma=\left\{\tau_{1}, \tau_{2}, \ldots, \tau_{n}\right\}$ of $n$ periodic real-time tasks, where task $\tau_{i}$ is modeled by the tuple $\tau_{i}=\left(T_{i}, D_{i}, E_{i}\right)$. The elements of the tuple are defined as follows: $T_{i}$ is the period of $\tau_{i}, D_{i}$ is its deadline $\left(D_{i} \leq T_{i}\right)$, and $E_{i}$ is the execution time under fault-free conditions.

Let the checkpointing cost be $C$. We make the following assumptions related to task execution and fault arrivals: (i) the task set $\Gamma$ is scheduled using fixed-priority methods such as the rate-monotonic scheme [7]; (ii) the task set $\Gamma$ is schedulable under fault free conditions; (iii) the priority of tasks are in decreasing order of the index $i$, i.e., task $\tau_{i}$ has higher priority than task $\tau_{j}$ if $i<j$; (iv) each instance of the task is released at the beginning of the period; (v) the checkpointing intervals are equal corresponding to the same task; (vi) the times for rollback and state restoration are zero; (vii) faults are detected as soon as they occur; (viii) no faults occur during checkpointing and rollback recovery.

In [13], a feasibility analysis is provided under the assumption that two successive faults arrive with a minimum inter-arrival time $T_{F}$. This implies that the time between the occurrences of two faults is at least $T_{F}$. This assumption is not practical for realistic applications, where the fault occurrence can be bursty or memoryless. For example, it is difficult to obtain a minimum inter-arrival time for a typical Poisson arrival process. Therefore, we focus here on tolerating up to a given number of faults during task execution. No additional assumption is made.

Since the task set is periodic, the total execution time can be very high if we consider a large number of periods. We therefore need to identify an appropriate $k$-fault-tolerant condition for shorter time duration. Here we provide two solutions corresponding to two different faulttolerance requirements. One is to tolerate $k$ faults for each job, termed as job-oriented fault tolerance; the other is to tolerate $k$ faults within a hyperperiod (defined as the least common multiple of all the task periods [7]), termed as hyperperiod-oriented fault tolerance. In practical situations, the choice of an appropriate fault tolerance criterion can be made based on the needs of the real-time application.

We first consider the case of a single job. Suppose $m$ checkpoints are inserted equidistantly during the computation time to tolerate $k$ faults in one job. The worst-case response time $R$ for the job is composed of three terms: the task execution time $E$, the checkpointing cost $m C$, and the recovery cost $k E /(m+1)$, i.e., $R=E+m C+k E /(m+1)$.

To satisfy the deadline constraint, we must have $E+m C+k E /(m+1) \leq D$.

Let $f(m)=E+m C+k E /(m+1)-D$. The minimum value of $f(m)$ is obtained for $m$ $=m_{0}=\sqrt{k E / C}-1$. Since $m$ is a non-negative integer, we have $m_{0}=\lceil\max (\sqrt{k E / C}-1,0)\rceil$.

If $f\left(m_{0}\right) \leq 0$, there exists equidistant checkpointing schemes for $k$-fault-tolerance, and the response time is minimum when $m_{0}$ checkpoints are inserted. If $f\left(m_{0}\right)>0$, then no equidistant checkpointing schemes exists for tolerating up to $k$ faults.

\subsection{Job-oriented fault tolerance: tolerating $\boldsymbol{k}$ faults in each job}

In this case, we require that the task set can meet the deadline requirement under the condition that at most $k$ faults occur during the execution of each job. The feasibility analysis is based on the time-demand analysis for fixed-priority scheduling [7]. The steps in the analysis are as following: 
(1) Compute the response time $R_{i}$ for $\tau_{i}$ according to the equation below (the right hand is defined as the time-demand function): $R_{i}=E_{i}+\sum_{h=1}^{i-1}\left\lceil R_{i} / T_{h}\right] E_{h}$. Here $T_{h}$ and $E_{h}$ are the period and the execution time of a task $\tau_{h}$ with higher priority than $\tau_{i}$. This equation can be solved by forming a recurrence relation:

$R_{i}^{(j+1)}=E_{i}+\sum_{h=1}^{i-1}\left[R_{i}^{(j)} / T_{h}\right] E_{h}$

(2) The iteration is terminated either when $R_{i}^{(j+1)}=R_{i}^{(j)}$ and $R_{i}^{(j)} \leq D_{i}$ for some $i$ or when $R_{i}{ }^{(j+1)}>D_{i}$, whichever occurs sooner. In the former case, $\tau_{i}$ is schedulable; in the later case, $\tau_{i}$ is not schedulable.

According to [7], the time complexity of the time-demand analysis for each task is $O(n W)$, where $W$ is the ratio of the largest period to the smallest period.

Under faulty conditions, the additional time due to checkpointing and recovery should be incorporated. When there are $m_{j}$ equidistant checkpoints for each instance of $\tau_{j}$, we have:

$$
R_{i}=\left(E_{i}+m_{i} C+k E_{i} /\left(m_{i}+1\right)\right)+\sum_{h=1}^{i-1}\left\lceil R_{i} / T_{h}\right]\left(E_{h}+m_{h} C+k E_{h} /\left(m_{h}+1\right)\right)
$$

To minimize all response times, we must have: $m_{i}{ }^{*}=\left\lceil\max \left(\sqrt{k E_{i} / C}-1,0\right)\right\rceil \quad(1 \leq i \leq n)$. Then we can employ the recurrence equation as follows:

$$
R_{i}{ }^{(j+1)}=\left(E_{i}+m_{i}{ }^{*} C+k \frac{E_{i}}{m_{i}{ }^{*}+1}\right)+\sum_{h=1}^{i-1}\left\lceil\frac{R_{i}{ }^{(j)}}{T_{h}}\right]\left(E_{h}+m_{h}{ }^{*} C+k \frac{E_{h}}{m_{h}{ }^{*}+1}\right) .
$$

When $R_{i}^{(j+1)}=R_{i}^{(j)}$ and $R_{i}^{(j)} \leq D_{i}$ for some $j, \tau_{i}$ is schedulable; when $R_{i}^{(j+1)}>D_{i}, \tau_{i}$ is not schedulable.

\subsection{Hyperperiod-oriented fault tolerance: tolerating $k$ faults in a hyperperiod}

In [13], an algorithm is presented to determine the checkpointing interval under the assumption that two successive faults arrive with a minimum inter-arrival time $T_{F}$. Let $F_{j}, 1 \leq j \leq i$, be the extra computation time needed by $\tau_{j}, 1 \leq j \leq i$, if one fault occurs during the execution. When there are $m_{j}$ equidistant checkpoints for $\tau_{j}$, the response time $R_{i}$ for $\tau_{i}$ is expressed as follows in [13]:

$R_{i}=\left(E_{i}+m_{i} C\right)+\sum_{h=1}^{i-1}\left\lceil R_{i} / T_{h}\right\rceil\left(E_{h}+m_{h} C\right)+\left\lceil R_{i} / T_{F}\right\rceil \max _{1 \leq j \leq i}\left\{F_{j}\right\}$, where $F_{j}=E_{j} /\left(m_{j}+1\right)$.

The checkpoint is examined starting from high-priority tasks to low-priority tasks. For each task $\tau_{j}$, the algorithm tries to reduce the response time by reducing the maximum additional computation time, i.e., $\max _{1 \leq j \leq i}\left\{F_{j}\right\}$. The details of the steps in [13] are as follows:

(1) Initially $m_{i}=0$ for $1 \leq i \leq n$.

(2) Starting from the highest-priority task $\tau_{1}$, calculate the minimum number of checkpoints $m_{1}$ required to make it schedulable.

(3) In decreasing order of task priorities, calculate the response time $R_{i}$ of task $\tau_{i}$. If $R_{i} \leq D_{i}$, move to the next task; otherwise $R_{i}$ need to be reduced further. The only way to reduce $R_{i}$ is to add more checkpoints to decrease the re-execution time caused by faults, i.e., $F_{j}$, for $1 \leq j \leq i$. In fact, the parameter $\max _{1 \leq j \leq i}\left\{F_{j}\right\}$ is relevant here and should be reduced. Thus the task $\tau^{*}$ that contributes the most to the task re-execution time is found and one more checkpoint is added to $\tau^{*}$. Then $R_{i}$ is recalculated. This process is repeated until either $R_{i} \leq D_{i}$ or the deadline $D_{i}$ is exceeded. 
This algorithm is based on the restrictive assumption that two successive faults arrive with a minimum inter-arrival time $T_{F}$. In addition, while the schedulability test in [13] provides useful guidelines on task schedulability in the presence of faults, a drawback of this work is that two key issues that affect schedulability have not been addressed.

1. Checkpoints are added to the higher-priority tasks in certain iterations in order to satisfy deadline constraints for all the tasks. These higher-priority tasks, however, have met their deadline in earlier iterations. The addition of more checkpoints to them inevitably changes their response times. As a result, it is necessary to trace back to re-calculate their response times and adjust their checkpoints. This issue has not been addressed in [13].

2. It is necessary to determine a bound on the number of checkpoints beyond which the addition of checkpoints does not improve schedulability. In another words, we need a criterion that can declare a task set to be not schedulable with a given number of checkpoints even though an arbitrary number of additional checkpoints can be added. In [13], the schedulability test concludes that $\tau_{i}$ is not schedulable once $R_{i}$ increases during the addition of checkpoints. However, this does not always hold. We present a counterexample below.

Example 1: Consider two tasks $\tau_{1}=(100,18,7.999)$ and $\tau_{2}=(101,21,8)$, and let $T_{F}=102, C$ $=0.1$. We follow the steps from [13] as below:

(1) Initially $m_{1}=m_{2}=0$, and $F_{1}=7.999, F_{2}=8$;

(2) Next $\tau_{1}$ is examined: $R_{1}=15.998<18$. No checkpoints are needed for $\tau_{1}$. Thus $m_{1}=m_{2}=0$.

(3) Next $\tau_{2}$ is examined: $R_{2}=23.999>21$. Since $F_{2}>F_{1}$, one checkpoint is added to $\tau_{2}$, thus $m_{1}$ $=0$ and $m_{2}=1$. Then $F_{1}=7.999, F_{2}=4$ and $\max _{1 \leq j \leq 2}\left\{F_{j}\right\}=7.999$. We recalculate the response time $R_{2}=24.098>23.999$. According to [13], $\tau_{2}$ is not schedulable. However, this is not correct. We continue the above step and find $F_{1}>F_{2}$, then one more checkpoint is added to $\tau_{1}$; as a result $m_{1}=1, m_{2}=1$. Then $F_{1}=7.999 /(1+1)=3.9995, F_{2}=4$, and $\max _{1 \leq j \leq 2}\left\{F_{j}\right\}=4$. We recalculate the response time of $\tau_{1}$ and $\tau_{2}: R_{1}=12.0985<18$ and $R_{2}=20.199<21$, which means both tasks are schedulable.

We require here that the tasks meet their deadlines under the condition that at most $k$ faults occur during a hyperperiod. Based on the schedulability test in [13], we solve the two aforementioned problems as follows.

The response time $R_{i}$ for $\tau_{i}$ is expressed as:

$$
R_{i}=\left(E_{i}+m_{i} C\right)+\sum_{h=1}^{i-1}\left\lceil R_{i} / T_{h}\right\rceil\left(E_{h}+m_{h} C\right)+k \max _{1 \leq j \leq i}\left\{F_{j}\right\}, \text { where } F_{j}=E_{j} /\left(m_{j}+1\right) .
$$

The first problem can be solved using a recursive method. Any time we increase the number of checkpoints for a task, all the lower-priority tasks need to be re-examined. The second problem is more complicated since the response time $R_{i}$ for task $\tau_{i}$ does not decreasing monotonically when more checkpoints are added to higher-priority tasks. Suppose that in $\max _{1 \leq j \leq i}\left\{F_{j}\right\}$, we find task $\tau_{h_{1}}$ contributes the most to the response time $R_{i}$, and add one more checkpoint to $\tau_{h_{1}}$. After recalculating $R_{i}$, we might find that $R_{i}$ has increased. In this situation, we cannot simply claim the task is not schedulable, as has been shown in Example 1.

We solve the second problem by determining a bound on the number of checkpoints such that if the task set cannot be made schedulable using this number of checkpoints, it cannot be scheduled by adding more checkpoints. Both the checkpointing cost and the timing constraints must be taken into account.

(1) Analysis of a bound based on checkpointing tradeoffs

The effect of adding more checkpoints is two-fold. First it increases the execution time due to the checkpoint cost, which runs contrary to the goal of reducing the response time. On the other hand, it decreases re-execution due to a fault, which helps in reducing the response time. Suppose the task execution time is $E$ and $m$ checkpoints have already been added. If another 
checkpoint is now added, the reduction of re-execution time under the $k$-fault-tolerance requirement is simply $k E /(m+1)-k E /(m+2)=k E /[(m+1)(m+2)]$. We combine the two impacts of checkpointing on the re-execution time to define the tradeoff function $\operatorname{tr}(m)$ as follows: $\operatorname{tr}(m)=C-k E /[(m+1)(m+2)]$.

If $\operatorname{tr}(m)<0$, then adding one more checkpoint can potentially reduce the response time; otherwise, it is not helpful since it increases the task re-execution time due to the $k$ faults.

For each task $\tau_{i}$ with $m_{i}$ checkpoints, we can calculate the tradeoff function $\operatorname{tr}_{i}\left(m_{i}\right)$. Solving for $\operatorname{tr}_{i}\left(m_{i}{ }^{\prime}\right)=0$, we get: $m_{i}{ }^{\prime}=\left(-3+\sqrt{1+4 k E_{i} / C}\right) / 2$ for $1 \leq i \leq n$. Since $m_{i}{ }^{\prime} \geq 0$, we further express it as: $m_{i}{ }^{\prime}=\max \left(\left\lfloor\left(-3+\sqrt{1+4 k E_{i} / C}\right) / 2\right\rfloor, 0\right)$ for $1 \leq i \leq n$. This gives an upper bound on the number of checkpoints, which is based on the tradeoff function.

(2) Analysis of a bound based on timing constraints

Under fault-free conditions, the response time $R_{i}{ }^{0}$ for task $\tau_{i}$ can be easily obtained. After incorporating the checkpointing cost and timing constraints, we have: $R_{i}{ }^{0}+m_{i} C \leq D_{i}$, which implies that $m_{i} \leq\left(D_{i}-R_{i}{ }^{0}\right) / C$. Let $m_{i}{ }^{\#}=\left\lfloor\left(D_{i}-R_{i}{ }^{0}\right) / C\right\rfloor$.

Combining the two bounds, we define $m_{i}{ }^{*}=\min \left(m_{i}{ }^{\prime}, m_{i}{ }^{*}\right)$ for $1 \leq i \leq n$. Then $m_{i}{ }^{*}$ is a tighter upper bound on the number of checkpoints required to make $\tau_{i}$ schedulable.

A checkpointing algorithm $A D V$ - $C P$ for off-line feasibility analysis is described in Figure 1 (a), which takes as an input parameter the real-time task set $\Gamma$. Line 1 initializes the parameters. The number of all checkpoints is set to 0 . The bounds for all tasks are calculated. All tasks are initially set unschedulable. Line 2 calls the recursive checkpointing subroutine $C P$ to add checkpoints from $\tau_{1}$ to $\tau_{n}$.

The recursive checkpointing procedure $C P(p, q)$ is described in Figure 1 (b), where $p$ and $q$ are the lowest and highest index for the task subset under consideration. Line 1 checks the deadline constraint to see if the current number of checkpoints can make the task subset schedulable. Line 2 checks to see if the bounds for the task subset are exceeded. If so, the whole task set is unschedulable and the recursive checkpointing should be exited. Line 3 further improves the feasibility of tasks from $\tau_{p}$ to $\tau_{q}$. Line 3.1 calculates $R_{j}$. If the deadline cannot be met for $\tau_{j}$ using the current number of checkpoints, Line 3.2 adds more checkpoints to higherpriority tasks or to $\tau_{j}$ itself. Line 3.2.1 finds the task $\tau_{h}$ that contributes most to the task reexecution time. Line 3.2.2 adds one more checkpoint to $\tau_{h}$, and Line 3.2.3 recalculates the reexecution time due to $\tau_{h}$. Finally, Line 3.2.4 employs the procedure $C P$ for tasks from $\tau_{h}$ to $\tau_{j}$.

The time complexity for the feasibility test and the checkpointing procedure can be analyzed as follows. The computation of $m_{i}{ }^{*}$ for all the tasks takes $O\left(n^{2} W\right)$ in the worst case. Each time a checkpoint is added, the response time for lower-priority tasks need to be recalculated. Hence the recursive execution of $C P(p, q)$ takes $O\left(n^{2} W\right) \sum_{i=1}^{n} m_{i}{ }^{*}$. Let $M^{*}=\sum_{i=1}^{n} m_{i}{ }^{*}$. Adding all the cost together, the total complexity is $O\left(n^{2} W M^{*}\right)$, which is only quadratic in the number of tasks $n$. Furthermore, we note that the complexity can be reduced if we can make $M^{*}$ as small as possible. That is why we combine both the tradeoff function and timing constraints to obtain a relatively tight bound for $m_{i}{ }^{*}$.

\subsection{Experimental results}

We compare the performance of our fault-tolerant schemes with the fault-oblivious schemes. Here we choose the rate-monotonic scheme (RM) as the fault--oblivious scheme. Our 


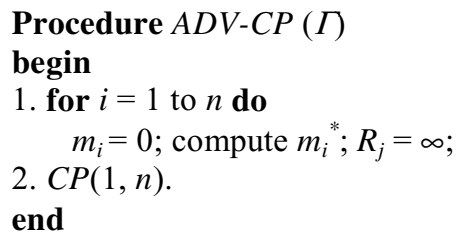

(a)

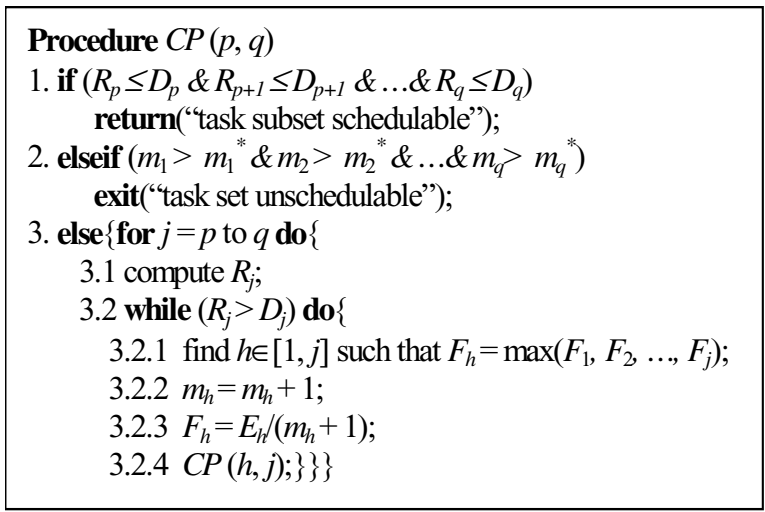

(b)

Figure 1. (a) Advanced checkpointing procedure (b) Recursive checkpointing procedure

goal here is to highlight the impact of fault occurrences on a fault-oblivious scheme. We use the following notation to refer to the two types of fault-tolerance schemes: JFT (job-oriented fault tolerance) and HFT (hyperperiod-oriented fault tolerance). We assume that RM simply reexecutes a job when a fault occurs.

3.3.1. JFT vs. RM: As pointed out in [7], a system of periodic preemptable tasks, each of whose relative deadline $D$ is equal to its period $T$, is schedulable on one processor according to the rate monotonic algorithm if and only if its total task utilization is equal to or less than 1 . In the presence of faults, since the re-execution takes extra time and the total task utilization will be increased accordingly, a task set that is schedulable under fault-free conditions may no longer be schedulable. Here we construct a task set whose total utilization is greater than 1 for RM under faulty conditions, and show that this task set is schedulable using JFT.

Suppose we are given three tasks $\tau_{1}=(12000,12000,2200), \tau_{2}=(18000,18000,3000)$ and $\tau_{3}=(24000,24000,4000)$. Let a single checkpoint take $C=50$. If only $k=1$ fault occurs during each job, the total utilization for RM is found to be 1.033. This implies that RM cannot schedule the task set when one or more faults occur during each job. However, the experiments show that JFT can tolerate up to 6 faults during each job. The number of checkpoints $\left(m_{1}, m_{2}, m_{3}\right)$ for $\tau_{1}$, $\tau_{2}$ and $\tau_{3}$ is shown in Table 1 (a).

To demonstrate the effect of checkpointing cost, we fix the value of $k$ and change the value of $C$ for the same task set used in Table 1 (a). The value of $C$ has no effect on the performance of RM, since it does not employ checkpointing. The results for $k=3$ are tabulated in Table 1 (b).

3.3.2. HFT vs. RM: We now show that HFT can schedule task sets in the presence of faults, even when RM fails to do so. Suppose we are given three tasks $\tau_{1}=(12000,12000,2200), \tau_{2}=$ $(18000,18000,3000)$ and $\tau_{3}=(24000,24000,4000)$, and three normalized processor speeds 1.0, 0.8 and 0.6. Let a single checkpoint take $C=50$. RM still cannot schedule this task set if there are any fault occurrences. On the other hand, HFT can tolerate more than 10 faults during a hyperperiod. The number of checkpoints $\left(m_{1}, m_{2}, m_{3}\right)$ for $\tau_{1}, \tau_{2}$ and $\tau_{3}$ is shown in Table 2 . The effect of checkpointing cost on the performance of HFT is not shown here due to lack of space.

\section{Conclusions}

We have presented feasibility-of-scheduling tests for checkpointing schemes that use a fixed checkpointing interval for hard real-time tasks. These tests provide the criteria under 


\begin{tabular}{|c|c|c|}
\hline$k$ & JFT: $\left(m_{1}, m_{2}, m_{3}\right)$ & RM \\
\hline 1 & $(6,7,8)$ & \\
\hline 2 & $(9,10,12)$ & \\
\hline 3 & $(11,13,15)$ & Infeasible \\
\hline 4 & $(13,15,17)$ & \\
\hline 5 & $(14,17,19)$ & \\
\hline 6 & $(16,18,21)$ & \\
\hline
\end{tabular}

(a)

\begin{tabular}{|c|c|c|}
\hline$C$ & JFT: $\left(m_{1}, m_{2}, m_{3}\right)$ & RM \\
\hline 50 & $(11,13,15)$ & \\
& \multirow{5}{*}{ Infeasible } \\
\hline 70 & $(10,12,14)$ & \\
\hline 80 & $(9,11,13)$ & \\
\hline 90 & $(9,10,12)$ & \\
\hline 100 & $(8,9,11)$ & \\
\hline
\end{tabular}

(b)

Table 1. JFT vs. RM: (a) Various $k$ (b) Various $C$

\begin{tabular}{|l|c|c|}
\hline$k$ & HFT: $\left(m_{1}, m_{2}, m_{3}\right)$ & RM \\
\hline 1 & $(0,0,1)$ & \\
\cline { 1 - 2 } 2 & $(1,1,2)$ & \multirow{3}{*}{ Infeasible } \\
\hline 4 & $(3,4,5)$ & \\
\hline 6 & $(4,6,8)$ & \\
\hline 8 & $(7,9,12)$ & \\
\hline 10 & $(10,14,19)$ & \\
\hline
\end{tabular}

Table 2. HFT vs. RM: various $k$

which checkpointing and rollback recovery can provide fault tolerance and real-time guarantees. We have considered two different fault arrival models: up to $k$ faults for a job, and up to $k$ faults for a hyperperiod. We have also presented techniques to determine a fixed checkpointing interval in an off-line manner.

\section{References}

[1] P. Pop, P. Eles and Z. Peng, "Schedulability analysis for systems with data and control dependencies", Proc. Euromicro RTS, pp. 201-208, 2000.

[2] J. Stankovic, "Misconceptions about real-time computing," IEEE Computer, vol. 21, pp. 10-19, October 1988.

[3] K. G. Shin and Y.-H. Lee, "Error detection process-Model, design and its impact on computer performance", IEEE Trans. Computers, vol. C-33, pp. 529-540, June 1984.

[4] K. M. Chandy, J. C. Browne, C. W. Dissly, and W. R. Uhrig, "Analytic Models for Rollback and Recovery Strategies in Data Base Systems”, IEEE Trans. Software Eng., vol. 1, pp. 100-110, March 1975.

[5] E. Dupont, M. Nicolaidis and P. Rohr, "Embedded robustness IPs for transient-error-free ICs", IEEE Design \& Test of Computers, vol. 19, pp. 54- 68, 2002.

[6] M. L. Bushnell and V. D. Agrawal, Essentials of Electronic Testing, Kluwer Academic Publishers, Norwell, MA, 2000 .

[7] J. W. Liu, Real-Time Systems, Prentice Hall, Upper Saddle River, NJ, 2000.

[8] Y. Zhang and K. Chakrabarty, "Energy-aware adaptive checkpointing in embedded real-time systems", Proc. Design, Automation and Test in Europe Conference, pp. 918-923, 2003.

[9] S. W. Kwak, B. J. Choi and B. K. Kim, "An optimal checkpointing-strategy for real-time control systems under transient faults”, IEEE Trans. Reliability, vol. 50, pp. 293-301, September 2001.

[10] A. Duda, "The effects of checkpointing on program execution time", Information Processing Letters, vol. 16, pp. 221-229, June 1983.

[11] H. Lee, H. Shin and S. Min, "Worst case timing requirement of real-time tasks with time redundancy", Proc. Real-Time Computing Systems and Applications, pp. 410-414, 1999.

[12] A. Ziv and J. Bruck, "An on-line algorithm for checkpoint placement", IEEE Trans. Computers, vol. 46, pp. 976-985, September 1997.

[13] S. Punnekkat, A. Burns and R. Davis, "Analysis of checkpointing for real-time systems", Real-Time Systems Journal, pp. 83-102, January 2001. 